University of Wollongong

Research Online

Australian Institute for Innovative Materials -

Papers

Australian Institute for Innovative Materials

2010

\title{
Magnetic properties of Bi2FeMnO6: a multiferroic material with double- perovskite structure
}

Yi Du

University of Wollongong, ydu@uow.edu.au

Zhenxiang Cheng

University of Wollongong, cheng@uow.edu.au

SX. Dou

University of Wollongong, shi@uow.edu.au

Xiaolin Wang

University of Wollongong, xiaolin@uow.edu.au

Hua Zhao

National Institute For Materials Science, hz739@uow.edu.au

See next page for additional authors

Follow this and additional works at: https://ro.uow.edu.au/aiimpapers

Part of the Engineering Commons, and the Physical Sciences and Mathematics Commons

Research Online is the open access institutional repository for the University of Wollongong. For further information contact the UOW Library: research-pubs@uow.edu.au 


\title{
Magnetic properties of Bi2FeMnO6: a multiferroic material with double- perovskite structure
}

\begin{abstract}
Single phase $\mathrm{Bi}_{2} \mathrm{FeMnO}_{6}$ was synthesized on $\mathrm{Si}$ substrates by an electrospray method. Three peaks were observed in the temperature dependence of magnetization curve, which is attributed to the inhomogeneous distribution of $\mathrm{Fe}^{3+}$ and $\mathrm{Mn}^{3+}$. The observed magnetic peaks at $150 \mathrm{~K}, 260 \mathrm{~K}$, and $440 \mathrm{~K}$ correspond to orderings of the ferrimagnetic Fe-O-Mn, and antiferromagnetic $\mathrm{Mn}-\mathrm{O}-\mathrm{Mn}$ and $\mathrm{Fe}-\mathrm{O}-\mathrm{Fe}$, respectively. Heat capacity measurements were carried out to confirm these magnetic transitions. The Debye temperature of $\mathrm{Bi}_{2} \mathrm{FeMnO}_{6}$ is $339 \mathrm{~K}$, calculated from Debye-Einstein fitting.
\end{abstract}

\section{Keywords}

Magnetic, properties, Bi2FeMnO6, multiferroic, material, double, perovskite, structure

\section{Disciplines}

Engineering | Physical Sciences and Mathematics

\section{Publication Details}

Du, Y, Cheng, ZX, Dou, SX, Wang, XL, Zhao, HY \& Kimura, H (2010), Magnetic properties of Bi2FeMnO6: a multiferroic material with double-perovskite structure, Applied Physics Letters, 97(12), pp. 1-3.

\section{Authors}

Yi Du, Zhenxiang Cheng, S X. Dou, Xiaolin Wang, Hua Zhao, and Hideo Kimura 


\title{
Magnetic properties of $\mathrm{Bi}_{2} \mathrm{FeMnO}_{6}$ : A multiferroic material with double-perovskite structure
}

\author{
Y. Du, ${ }^{1}$ Z. X. Cheng, ${ }^{1, a)}$ S. X. Dou, ${ }^{1}$ X. L. Wang, ${ }^{1, a)}$ H. Y. Zhao, ${ }^{2}$ and H. Kimura ${ }^{2}$ \\ ${ }^{1}$ Innovation Campus-AIIM Facility, Institute for Superconducting and Electronic Materials (ISEM), University \\ of Wollongong, Squires Way, Fairy Meadow, New South Wales 2519, Australia \\ ${ }^{2}$ National Institute for Materials Science, Sengen 1-2-1, Tsukuba, Japan
}

(Received 25 May 2010; accepted 17 August 2010; published online 21 September 2010)

\begin{abstract}
Single phase $\mathrm{Bi}_{2} \mathrm{FeMnO}_{6}$ was synthesized on $\mathrm{Si}$ substrates by an electrospray method. Three peaks were observed in the temperature dependence of magnetization curve, which is attributed to the inhomogeneous distribution of $\mathrm{Fe}^{3+}$ and $\mathrm{Mn}^{3+}$. The observed magnetic peaks at $150 \mathrm{~K}, 260 \mathrm{~K}$, and $440 \mathrm{~K}$ correspond to orderings of the ferrimagnetic $\mathrm{Fe}-\mathrm{O}-\mathrm{Mn}$, and antiferromagnetic $\mathrm{Mn}-\mathrm{O}-\mathrm{Mn}$ and $\mathrm{Fe}-\mathrm{O}-\mathrm{Fe}$, respectively. Heat capacity measurements were carried out to confirm these magnetic transitions. The Debye temperature of $\mathrm{Bi}_{2} \mathrm{FeMnO}_{6}$ is $339 \mathrm{~K}$, calculated from Debye-Einstein fitting. () 2010 American Institute of Physics. [doi:10.1063/1.3490221]
\end{abstract}

Multiferroic materials which display coexistence of ferromagnetism (FM) and ferroelectric (FE) polarization have drawn considerable attention due to their fundamental physical characteristics and potentially wide applications. ${ }^{1-4}$ Unfortunately, candidate multiferroic materials are very rare due to the mutual exclusivity of the origins of FM and electric polarization, in which FM needs transition metals with unpaired $3 d$ electrons and unfilled $3 d$ orbital, while FE polarization needs transition metals with filled $3 d$ orbital. Furthermore, the available multiferroic candidates, such as $\mathrm{BiFeO}_{3},{ }^{5-9} \mathrm{BiMnO}_{3},{ }^{10-12}$ and $\mathrm{DyFeO}_{3},{ }^{13}$ all have serious drawbacks, i.e., electric leakage or low temperature for appearance of multiferroic properties. Therefore, efforts to explore new candidate multiferroic materials are still highly desirable. Recently, a multiferroic material with doubleperovskite structure, $\mathrm{Bi}_{2} \mathrm{FeMnO}_{6}$, has been theoretically designed. ${ }^{14}$ It is expected that this material would show simultaneous magnetic ordering and electric polarization because it contains $\mathrm{Bi}^{3+}$ ions with unique $6 s^{2}$ lone-pair electrons, similar to the examples of other bismuth transition metal oxides with simple perovskite structure. However, only a few studies on this compound have been reported, possibly due to the difficulties in the fabrication of this metastable material through a conventional high temperature synthesis process. ${ }^{14}$ Therefore, reports on the detailed physical properties of this material have been awaited with great interest. In previous work, ${ }^{15}$ room temperature multiferroic properties were reported for $\mathrm{Nd}: \mathrm{BiFeO}_{3} / \mathrm{Bi}_{2} \mathrm{FeMnO}_{6}$ bilayer films fabricated by the pulsed laser deposition method, and FM and FE properties were observed. However, due to the appearance of $\mathrm{BiFeO}_{3}$, it is hard to subtract the intrinsic magnetic properties of $\mathrm{Bi}_{2} \mathrm{FeMnO}_{6}$. In this study, pure $\mathrm{Bi}_{2} \mathrm{FeMnO}$ samples were fabricated by an electrospray method. Its intrinsic magnetic properties have been observed.

Stoichiometric $\mathrm{Bi}\left(\mathrm{NO}_{3}\right)_{3} \cdot 5 \mathrm{H}_{2} \mathrm{O}$ and $\mathrm{Fe}\left(\mathrm{NO}_{3}\right)_{3} \cdot 9 \mathrm{H}_{2} \mathrm{O}$, and $\mathrm{NH}_{4} \mathrm{OH}$ were dissolved in 2-methoxyethanol, forming dark brown $0.005 \mathrm{M}$ precursor. $\mathrm{Bi}_{2} \mathrm{FeMnO}_{6}$ film was synthesized on a silicon substrate (heated up to $973 \mathrm{~K}$ ) by an electrospray method in a high voltage field $(20 \mathrm{kV})$ over $2 \mathrm{~h}$.

\footnotetext{
a) Authors to whom correspondence should be addressed. Electronic addresses: xiaolin@uow.edu.au and cheng@uow.edu.au.
}

After that, the sample was sintered at $1173 \mathrm{~K}$ for $1 \mathrm{~h}$. The sample was characterized by $\mathrm{x}$-ray diffraction (XRD), XRD Rietveld refinement, scanning electron microscopy (SEM) equipped with energy dispersive spectroscopy (EDS). $B\left(B^{\prime}\right)$-site ion valence states were identified on an X-ray photoelectron spectrometer (XPS). Magnetic and thermal properties were measured by a $14 \mathrm{~T}$ physical property measurement system (14T PPMS, Quantum Design) from 2 to $750 \mathrm{~K}$ in magnetic field up to $10 \mathrm{kOe}$.

Figure 1(a) shows the XRD pattern and refinement calculation results for $\mathrm{Bi}_{2} \mathrm{FeMnO}_{6}$. The XRD pattern of substrate has also been plotted in the figure. The sample is a well-crystallized single-phase film. $\mathrm{Bi}_{2} \mathrm{FeMnO}_{6}$ is a meta-
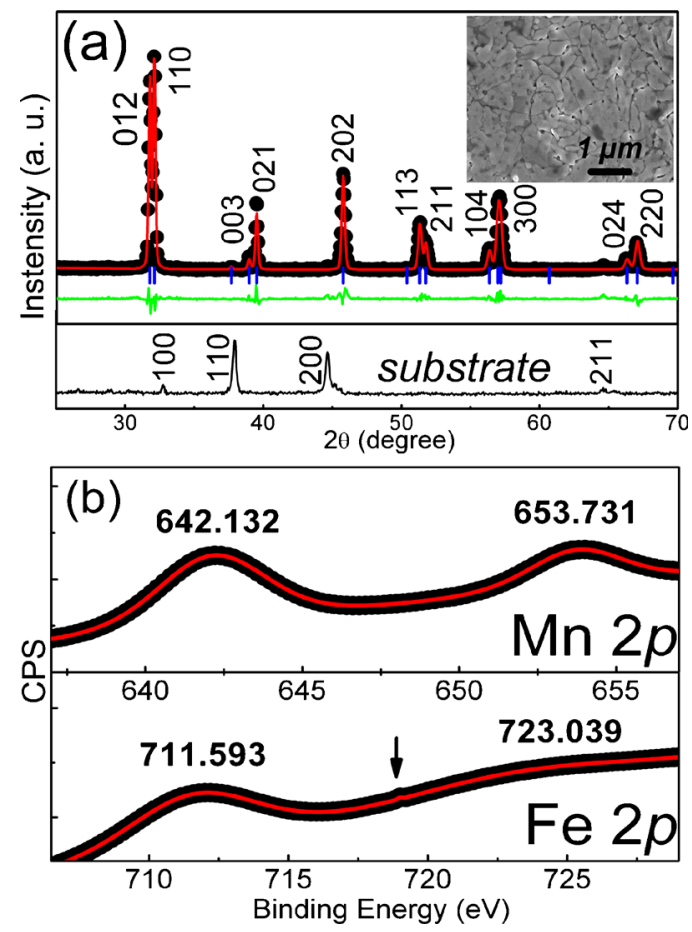

FIG. 1. (Color online) (a) XRD refinement calculation result for as-prepared $\mathrm{Bi}_{2} \mathrm{FeMnO}_{6}\left(R\right.$ factors: $\left.R_{\mathrm{P}}=8.1 \%, R_{\mathrm{WP}}=10.7 \%\right)$. The XRD pattern of the substrate has been plotted as a reference. The inset is the SEM image for double-perovskite $\mathrm{Bi}_{2} \mathrm{FeMnO}_{6}$ film. (b) XPS spectra of the Fe $2 p$ peaks and the $\mathrm{Mn} 2 p$ peaks for $\mathrm{Bi}_{2} \mathrm{FeMnO}_{6}$ film. 
stable compound due to the positive formation enthalpy, as determined by first principles calculations. ${ }^{14}$ It has been reported that its crystal structure varies from the $\operatorname{Pm} \overline{3} m$, to the $R 3 c$, to the $C 2$ space group, depending on the fabrication conditions. In this work, each of these structures has been adopted to refine the as-obtained XRD pattern. It was found that all the diffraction peaks except peaks from substrate can be indexed by using space group $R 3 c$ and a reliable refinement result could be achieved in contrast to the unreasonable deviations for refinement based on $P m \overline{3} m$ and $C 2$ groups. The refinement results show that $\mathrm{Bi}_{2} \mathrm{FeMnO}_{6}$ has a unit cell with lattice parameters $a=5.2575 \AA, b=5.2575 \AA$, and $c$ $=13.072 \AA$. The $\mathrm{FeO}_{6}$ and $\mathrm{MnO}_{6}$ polyhedra are elongated in the out-of-plane direction ( $c$ axis). The SEM image [Fig. 1(a) inset] indicates that the as-obtained sample is a polycrystalline granular film over the substrate. The thickness of film is around $550 \mathrm{~nm}$. EDS spectra confirm the presence of the constituent elements in the sample with a ratio of $\sim 1.97: 1: 1$ in the order of $\mathrm{Bi}, \mathrm{Fe}$, and $\mathrm{Mn}$, which is very close to the stoichiometry of $\mathrm{Bi}_{2} \mathrm{FeMnO}_{6}$. The slight deficiency of the bismuth will possibly lead to oxygen deficiency in order to keep charge balance in the compound which is very common for perovskite oxides, instead of formation of impurity phase which has been excluded by XRD result. It is difficult to have a conclusion of element distribution from EDS mapping results because the atomic distribution of $\mathrm{Mn}$ and $\mathrm{Fe}$ is out of the ability of EDS. In the stoichiometric $\mathrm{Bi}_{2} \mathrm{FeMnO}_{6}$, the average valence state of the $B\left(B^{\prime}\right)$-site cations is +3 . Mn tends to exhibit multiple valences in many compounds with distorted perovskite structure, ${ }^{16-18}$ which results in nonstoichiometric oxygen content and also reversely is a result of oxygen deficiency. In order to determine the valences of $\mathrm{Mn}$ and $\mathrm{Fe}$ ions in our sample, XPS measurements have been carried out, as shown in Fig. 1(b). In Mn $2 p$ XPS result, only two main peaks of the $2 p 1 / 2(653.731 \mathrm{eV})$ and $2 p 3 / 2$ $(642.132 \mathrm{eV})$, which are corresponding to $\mathrm{Mn}^{3+}$ ions, have been observed. It is known that the $\mathrm{Fe} 2 p$ photoelectron peaks $(2 p 1 / 2$ at $723.0389 \mathrm{eV}$ and $2 p 3 / 2$ at $711.593 \mathrm{eV})$ from oxidized iron are associated with satellite peaks, which is important for identifying the chemical states. The satellite peak was found $8 \mathrm{eV}$ above the $2 p 3 / 2$ principal peak, in $\mathrm{Bi}_{2} \mathrm{FeMnO}_{6}$, which indicates the valence of $\mathrm{Fe}$ ions is +3 . These results confirm that $\mathrm{Mn}$ and $\mathrm{Fe}$ ions in $\mathrm{Bi}_{2} \mathrm{FeMnO}_{6}$ film only present valence of +3 .

Figure 2(a) displays the temperature dependence of the zero-field-cooled and field-cooled magnetic susceptibility $\chi(T)$, measured in a magnetic field of $H=1000$ Oe from 5 to $750 \mathrm{~K}$ for $\mathrm{Bi}_{2} \mathrm{FeMnO}_{6}$. From high temperature to low temperature, three magnetic peaks were observed, at $440 \mathrm{~K}\left(\mathrm{~T}_{1}\right)$, $260 \mathrm{~K}\left(\mathrm{~T}_{2}\right)$, and $150 \mathrm{~K}\left(\mathrm{~T}_{3}\right)$. Curie-Weiss fitting of the $1 / \chi-T$ curve from 500 to $750 \mathrm{~K}$, when the sample is in a paramagnetic state, was carried out as shown in the inset. The effective moment $\left(\mu_{\mathrm{eff}}\right)$ on the $B\left(B^{\prime}\right)$-site was calculated to be $\mu_{\text {eff }}=7.5 \mu_{\mathrm{B}}$, where $\mu_{\mathrm{B}}$ is the Bohr magneton. This result indicates that both $\mathrm{Fe}^{3+}$ and $\mathrm{Mn}^{3+}$ present high spin state $\left(H S, t_{2 g}^{3} e_{g}^{2}\right.$ for $\mathrm{Fe}^{3+}$ and $t_{2 g}^{3} e_{g}^{1}$ for $\left.\mathrm{Mn}^{3+}\right)$ electron configurations. The very high Curie-Weiss temperature, $\theta_{P}=-1020 \mathrm{~K}$ indicates that $\mathrm{Bi}_{2} \mathrm{FeMnO}_{6}$ undergoes a strong antiferromagnetic (AFM) transition at $\mathrm{T}_{1}$. According to the Goodenough-Kanamori rules for $180^{\circ}$ superexchange coupling, ${ }^{19}$ dissimilar ions of $\mathrm{Fe}^{3+}-\mathrm{Mn}^{3+}$ with both ions in the high spin state with a half-filled $e_{g}$ orbital and one empty
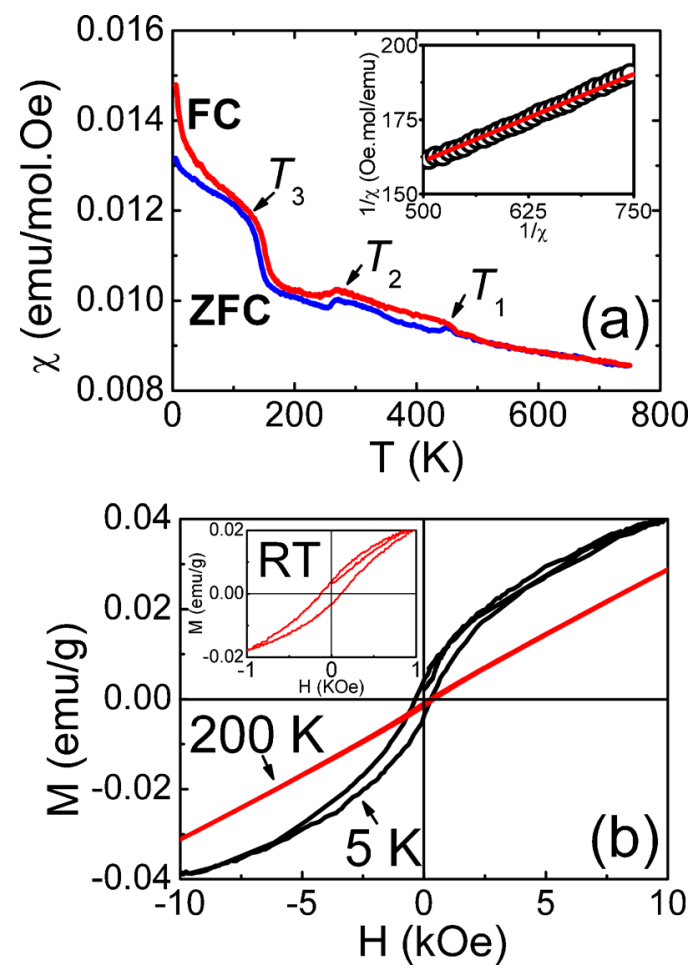

FIG. 2. (Color online) (a) Magnetization as a function of temperature ( $5 \mathrm{~K}<T<750 \mathrm{~K}$ ); the solid line in the inset is the Curie-Weiss fitting for the $M-T$ curve from 500 to $750 \mathrm{~K}$. (b) Magnetization as a function of magnetic field at different temperatures; the inset is the $M-H$ hysteresis loop observed at room temperature. The magnetic contribution from the substrate has been subtracted from all the data.

$e_{g}$ orbital, most likely will result in antiparallel spin ordering. It has been reported that distribution of $\mathrm{Fe}^{3+}$ and $\mathrm{Mn}^{3+}$ ions is not homogeneous in such double-perovskite structures due to the very close ionic radii of $\mathrm{Mn}^{3+}$ and $\mathrm{Fe}^{3+} \cdot{ }^{18} \mathrm{As}$ a result, Fe-rich and Mn-rich clusters will form. Again, the Goodenough-Kanamori rules show that $\mathrm{Fe}-\mathrm{O}-\mathrm{Fe}$ in $\mathrm{TMO}_{6}$ octahedra, where TM is a transition metal, will be ordered antiferromagnetically through $180^{\circ}$ superexchange coupling. A strong AFM $e_{g}^{2}-\mathrm{O}-e_{g}^{2}(\mathrm{Fe}-\mathrm{O}-\mathrm{Fe})$ interaction is favored by virtual charge transfer due to the two half-filled $e_{g}$ orbitals of $H S \mathrm{Fe}^{3+}$. For $\mathrm{Mn}-\mathrm{O}-\mathrm{Mn}$, it can order antiferromagnetically through empty $d \mathrm{z}^{2}$ to $d \mathrm{z}^{2}$ and $d \mathrm{x}^{2}-\mathrm{y}^{2}$ to $d \mathrm{x}^{2}-\mathrm{y}^{2}$ orbits interactions both via oxygen $2 p$ orbital and order ferromagnetically through superexchange mechanism between Mn halffilled $d \mathrm{z}^{2}$ and empty $d \mathrm{x}^{2}-\mathrm{y}^{2}$ orbitals via oxygen $2 p$ orbital in case of orbit ordering in $\mathrm{BiMnO}_{3} .{ }^{14}$ However, no evidence of orbital ordering can be found in the present data. Furthermore, based on the small magnetic moment observed in $M-H$ loop, we conclude that $\mathrm{Mn}-\mathrm{O}-\mathrm{Mn}$ is antiferromagnetic ordered in $\mathrm{Bi}_{2} \mathrm{FeMnO}_{6}$. Because only one unpaired electron occupies the $e_{g}$ orbital, $\mathrm{Mn}-\mathrm{O}-\mathrm{Mn}\left(e_{g}^{1} \mathrm{O}-e_{g}^{1}\right)$ ordering is much weaker than $\mathrm{Fe}-\mathrm{O}-\mathrm{Fe}$ ordering. Hence, the temperature of $\mathrm{Fe}-\mathrm{O}-\mathrm{Fe}$ ordering will be higher than that of $\mathrm{Mn}$ $\mathrm{O}-\mathrm{Mn}$ ordering. Based on the above reasons, the origins for the magnetic transitions at temperatures of $440 \mathrm{~K}, 260 \mathrm{~K}$, and $150 \mathrm{~K}$ are attributed to $\mathrm{Fe}-\mathrm{O}-\mathrm{Fe}, \mathrm{Mn}-\mathrm{O}-\mathrm{Mn}$, and $\mathrm{Fe}-\mathrm{O}-\mathrm{Mn}$ orderings, respectively.

To further confirm the above analysis, magnetization $(M)$ as a function of magnetic field $(H)$ was measured at various temperatures. At $300 \mathrm{~K}$, an unsaturated hysteresis loop with coercive field of 118 Oe is observed with AFM and FM components in a magnetic field of $1 \mathrm{kOe}$, as shown in 


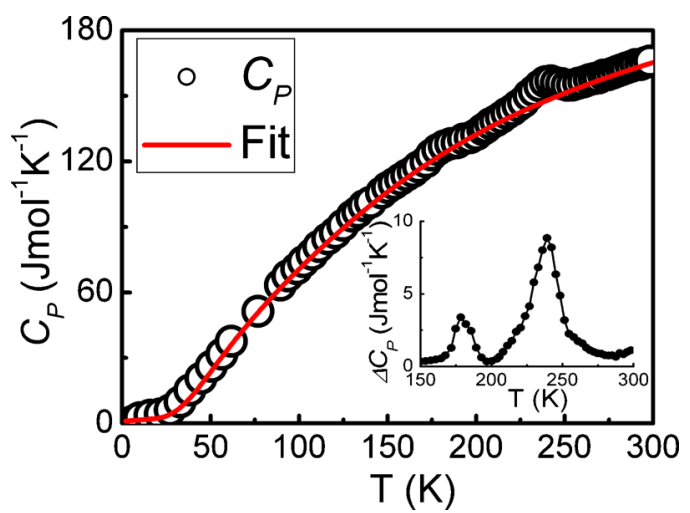

FIG. 3. (Color online) Heat capacity as a function of temperature from 2 to $300 \mathrm{~K}$ (symbols show the experimental data, and solid line is the fit). The inset is the excess magnetic heat capacity extracted from the total heat capacity by subtracting a smooth background using Debye-Einstein fitting.

the Fig. 2(b) inset. The magnetization is estimated to be $0.42 \mu_{\mathrm{B}} /$ f.u. at $1 \mathrm{kOe}$. The FM component is possibly attributable to a canted Fe moment arrangement. At $200 \mathrm{~K}$, hysteresis behavior still remains in the $M-H$ curve, with a coercive field of 82 Oe. However, the total behavior of $M-H$ is dominated by a significant AFM contribution. At $5 \mathrm{~K}$, a clear hysteresis loop with coercive field of 330 Oe and much reduced linear behavior is observed. The magnetic moment is estimated to be $4.48 \mu_{\mathrm{B}} / \mathrm{f}$.u. at $10 \mathrm{kOe}$, which is much smaller than the theoretical value of $9 \mu_{\mathrm{B}} /$ f.u. if all $\mathrm{Fe}^{3+}$ and $\mathrm{Mn}^{3+}$ ions are ordered ferromagnetically on the $B\left(B^{\prime}\right)$-site. Therefore, the magnetic transition at $\mathrm{T}_{3}$ attributes to $\mathrm{Fe}-\mathrm{Mn}$ ferrimagnetic ordering transition due to the small magnetic moment of the materials at low temperature.

The temperature dependence $(2 \mathrm{~K}<T<300 \mathrm{~K})$ of the zero magnetic field heat capacity, $C_{p}$, is shown in Fig. 3 for $\mathrm{Bi}_{2} \mathrm{FeMnO}_{6}$. The overall shape of $C_{p}(T)$ follows the DulongPetit law, that is, the high temperature limiting value is consistent with the classical value of $3 R$ per atomic site in the formula unit, where $R$ is the molar gas constant. There are two clear $\lambda$-shaped anomaly peaks with peak temperatures of 242 and $175 \mathrm{~K}$ in the $C_{p}(T)$ curve, corresponding to the magnetic transitions at $\mathrm{T}_{2}$ and $\mathrm{T}_{3}$. Since no nonmagnetic analogs are available for this compound, the nonmagnetic contribution can be calculated by a combination of Debye and Einstein equations, ${ }^{20}$

$$
\begin{aligned}
C_{p}^{n m}= & N k_{\mathrm{B}}\left\{\frac{9}{1-\eta_{\mathrm{D}} T}\left(\frac{T}{\theta_{\mathrm{D}}}\right)^{3} \int_{0}^{\theta_{\mathrm{D} / T}} \frac{x^{2} \exp (x)}{[\exp (x)-1]^{2}} d x\right. \\
& \left.+\sum_{i=1}^{n} \frac{1}{1-\eta_{\mathrm{E}} T} \frac{\left(\theta_{\mathrm{Ei}} / T\right)^{2} \exp \left(\theta_{\mathrm{Ei}} / T\right)}{\left[\exp \left(\theta_{\mathrm{Ei}} / T\right)-1\right]^{2}}\right\},
\end{aligned}
$$

where $\eta_{\mathrm{D}}$ and $\eta_{\mathrm{E}}$ are the respective Debye and Einstein anharmonicity coefficients, $\theta_{\mathrm{D}}$ is the Debye temperature, $\theta_{\mathrm{E}}$ is the Einstein temperature, $k_{\mathrm{B}}$ is the Boltzmann constant, and $N$ is mole number of the sample. The best fit was obtained by using two Einstein terms with an equivalent value for $\eta_{\mathrm{D}}$ and $\eta_{\mathrm{E}}$. The fit is plotted in Fig. 3 as a solid line, which is in good agreement with the experimental data in the high temperature range. The parameters with the best fit were found to be $\eta_{\mathrm{D}}=0.0011 \mathrm{~K}^{-1}, \eta_{\mathrm{E}}=0.0011 \mathrm{~K}^{-1}, \theta_{\mathrm{D}}=339 \mathrm{~K}, \theta_{\mathrm{E} 1}$ $=255 \mathrm{~K}$, and $\theta_{\mathrm{E} 2}=613 \mathrm{~K}$. The Debye temperature is comparable with those obtained for $\mathrm{BiFeO}_{3}(340 \mathrm{~K})$ and $\mathrm{BiMnO}_{3}$ $(290 \mathrm{~K}),{ }^{21-23}$ indicating the similar thermodynamic proper- ties of these compounds. The magnetic contribution to the heat capacity, $\Delta C_{p}(T)$, therefore can be obtained by subtracting the nonmagnetic contribution from $C_{p}(T)$, as shown in the Fig. 3 inset. The high and broad peak at $T_{2}$ reflects the AFM Mn-O-Mn interaction. The peak at $175 \mathrm{~K}$ is associated with ferrimagnetic $\mathrm{Fe}-\mathrm{Mn}$ ordering. Furthermore, the observation of this peak excludes its origin from spin-glass behavior, which has been observed in other materials with competition between different magnetic orderings.

In conclusion, $\mathrm{Bi}_{2} \mathrm{FeMnO}_{6}$ with double-perovskite structure was synthesized by an electrospray method. Magnetic measurements show the existence of Mn-rich and Fe-rich clusters. Although the fabrication method, starting from a solution precursor with an atomic-level $\mathrm{Mn}$ and $\mathrm{Fe}$ homogeneous distribution, was expected to result in $\mathrm{Bi}_{2} \mathrm{FeMnO}_{6}$ with homogeneous distribution of $\mathrm{Mn}$ and $\mathrm{Fe}$, Mn-rich or Fe-rich clusters still appear due to their very close ionic radii and same charges. Therefore, it might be impossible to realize a one-by-one atomic arrangement of $\mathrm{Mn}$ and $\mathrm{Fe}$ in this material.

The authors thank the ARC for support through a Discovery Project and a Future Fellowship. Y. Du thanks Dr. Z. P. Guo and Mr. P. Zhang for help in using the electrospray system.

${ }^{1}$ B. K. Ponomarev, S. A. Ivanov, Y. F. Popov, V. D. Negrii, and B. S. Red'kin, Ferroelectrics 161, 43 (1994).

${ }^{2}$ H. Schmid, Ferroelectrics 162, 317 (1994).

${ }^{3}$ N. A. Hill, J. Phys. Chem. B 104, 6694 (2000).

${ }^{4}$ M. Gajek, M. Bibes, A. Barthélémy, K. Bouzehouane, S. Fusil, M. Varela, J. Fontcuberta, and A. Fert, Phys. Rev. B 72, 020406(R) (2005).

${ }^{5}$ Z. X. Cheng, X. L. Wang, S. X. Dou, K. Ozawa, and H. Kimura, Phys. Rev. B 77, 092101 (2008).

${ }^{6}$ J. B. Neaton, C. Ederer, U. V. Waghmare, N. A. Spaldin, and K. M. Rabe, Phys. Rev. B 71, 014113 (2005).

${ }^{7}$ J. Wang, J. B. Neaton, H. Zheng, V. Nagarajan, B. Ogale, B. Liu, D. Viehland, V. Vaithyanathan, D. G. Schlom, U. V. Waghmare, N. A. Spaldin, K. M. Rabe, M. Wuttig, and R. Ramesh, Science 299, 1719 (2003).

${ }^{8}$ Z. X. Cheng, X. L. Wang, H. Kimura, K. Ozawa, and S. X. Dou, Appl. Phys. Lett. 92, 092902 (2008).

${ }^{9}$ Y. Chu, Q. He, C. Yang, P. Yu, L. W. Martin, P. Shafer, and R. Ramesh, Nano Lett. 9, 1726 (2009).

${ }^{10}$ R. Schmidt, W. Eerenstein, and P. A. Midgley, Phys. Rev. B 79, 214107 (2009).

${ }^{11}$ T. Kimura, S. Kawamoto, I. Yamada, M. Azuma, M. Takano, and Y Tokura, Phys. Rev. B 67, 180401(R) (2003).

${ }^{12}$ R. Schmidt, W. Eerenstein, T. Winiecki, F. D. Morrison, and P. A. Midgley, Phys. Rev. B 75, 245111 (2007).

${ }^{13}$ Y. Tokunaga, S. Iguchi, T. Arima, and Y. Tokura, Phys. Rev. Lett. 101, 097205 (2008).

${ }^{14}$ L. Bi, A. R. Taussig, H. Kim, L. Wang, G. F. Dionne, D. Bono, K. Persson, G. D. Ceder, and C. A. Ross, Phys. Rev. B 78, 104106 (2008).

${ }^{15}$ H. Zhao, H. Kimura, Z. Cheng, X. Wang, and T. Nishida, Appl. Phys. Lett. 95, 232904 (2009).

${ }^{16}$ J. B. Goodenough, A. Wold, R. J. Arnott, and N. Menyuk, Phys. Rev. 124, 373 (1961).

${ }^{17}$ W. Bao, J. D. Axe, C. H. Chen, and S. Cheong, Phys. Rev. Lett. 78, 543 (1997).

${ }^{18}$ W. Tong, B. Zhang, S. Tan, and Y. Zhang, Phys. Rev. B 70, 014422 (2004)

${ }^{19}$ J. B. Goodenough, Magnetism and the Chemical Bond (Wiley, New York, 1963).

${ }^{20}$ C. A. Martin, J. Phys.: Condens. Matter 3, 5967 (1991).

${ }^{21}$ C. Blaauw and F. van der Woude, J. Phys. C 6, 1422 (1973).

${ }^{22}$ A. A. Belik and E. Takayama-Muromachi, Inorg. Chem. 45, 10224 (2006).

${ }^{23}$ See supplementary material at http://dx.doi.org/10.1063/1.3490221 for SEM, XPS, and EDS mapping results. 